\title{
ESTUDO COMPARATIVO DOS PROCEDIMENTOS PERCUTÂNEOS ORIENTADOS POR MÉTODOS DE IMAGEM NO TRATAMENTO DAS COLEÇÕES HEPÁTICAS
}

\author{
Giselle Guedes Netto de Mello ${ }^{1}$, Gustavo de Souza Portes Meirelles ${ }^{2}$, José Marcelo Amatuzzi \\ de Oliveira ${ }^{1}$, Sergio Ajzen ${ }^{3}$, Giuseppe D'Ippolito ${ }^{4}$, Jacob Szejnfeld ${ }^{5}$
}

Resumo OBJETIVO: Verificar e comparar a eficácia da punção aspirativa e da drenagem percutânea, orientadas por métodos de imagem, no tratamento das coleções hepáticas. MATERIAIS E MÉTODOS: Foram encaminhados, ao nosso setor, 52 pacientes para tratamento percutâneo de coleções abdominais. Destes, 17 apresentavam coleções hepáticas, sendo 13/17 não-complexas e 4/17, complexas (multiloculadas, múltiplas ou associadas a fístulas). Foram submetidas a punção aspirativa $7 / 17$ coleções $(41,2 \%)$ e a drenagem percutânea, 10/17 (58,8\%). Considerou-se como sucesso do método a resolução completa da coleção, com melhora clínica e laboratorial do paciente. RESULTADOS: 0 sucesso do procedimento ocorreu em $82,4 \%$ dos casos. Nas coleções submetidas exclusivamente a punção aspirativa, obteve-se sucesso em $57,1 \%$, enquanto nas submetidas a drenagem percutânea o sucesso foi de $100 \%$. 0 índice de sucesso no grupo submetido a punção aspirativa foi de 75\% nas coleções menores que $100 \mathrm{ml}$, e de 33,3\% naquelas entre 100 e $250 \mathrm{ml}$. Houve sucesso com a punção aspirativa em $75 \%$ das coleções não-complexas e em $25 \%$ das complexas. CONCLUSÃO: A drenagem percutânea é mais eficaz que a punção aspirativa no tratamento das coleções hepáticas. A punção aspirativa talvez possa ser empregada como alternativa válida nas coleções de menor volume e não-complexas.

Unitermos: Coleções hepáticas. Punção aspirativa. Drenagem percutânea.

\begin{abstract}
A comparative study of image-guided percutaneous procedures for the treatment of liver abscesses. OBJECTIVE: To determine and compare the efficacy of percutaneous needle aspiration and percutaneous catheter drainage, both guided by imaging methods, for the treatment of liver abscesses. MATERIALS AND METHODS: From 52 patients referred to our service for percutaneous treatment of abdominal abscesses, 17 presented liver abscesses, 13 of which were considered noncomplex and four were considered complex (multiloculated, multiple or associated to fistulas). Percutaneous needle aspiration was performed in 7/17 patients and 10/17 patients were submitted to percutaneous catheter drainage. The method used was considered successful when there was complete abscess resolution with both clinical and laboratorial improvement. RESULTS: The procedures were successful in $\mathbf{8 2 . 4 \%}$ of all cases. In the group submitted to percutaneous needle aspiration the rate of success was $57.1 \%$ and in the group submitted to percutaneous catheter drainage the rate of success was $100 \%$. Successful treatment was achieved in $75 \%$ of the patients submitted to percutaneous needle aspiration for abscesses smaller than $100 \mathrm{ml}$, but in only $33.3 \%$ of the patients with abscesses between 100 and $250 \mathrm{ml}$. There was complete resolution of the abscesses with percutaneous needle aspiration in $75 \%$ of the simple abscesses and in $25 \%$ of the complex abscesses. CONCLUSION: Percutaneous catheter drainage is more effective than percutaneous needle aspiration for the treatment of liver abscesses. Needle aspiration may probably be used as a valid alternative for smaller and noncomplex abscesses.

Key words: Liver abscesses. Percutaneous needle aspiration. Percutaneous catheter drainage.
\end{abstract}

* Trabalho realizado no Departamento de Diagnóstico por Imagem da Universidade Federal de São Paulo/Escola Paulista de Medicina (Unifesp/EPM), São Paulo, SP.

1. Médicos do Setor de Abdome do Departamento de Diagnóstico por Imagem da Unifesp/EPM.

2. Médico Residente do Departamento de Diagnóstico por Imagem da Unifesp/EPM.

3. Professor Adjunto do Departamento de Diagnóstico por Imagem da Unifesp/EPM.

4. Professor Visitante do Departamento de Diagnóstico por Imagem da Unifesp/EPM.

5. Professor Livre-Docente, Chefe do Departamento de Diagnóstico por Imagem da Unifesp/EPM.

Endereço para correspondência: Dra. Giselle Guedes Netto de Mello. Rua Antônio Lucas de Oliveira, 27, Veleiros. São Paulo, SP, 04773-060.

Aceito para publicação em 1/9/2000.

\section{INTRODUÇÃO}

Os abscessos hepáticos são afecções graves, com índices de mortalidade que podem chegar a $100 \%$ quando não-drenados $^{(1,2)}$. O reconhecimento e o esvaziamento precoce desses abscessos são imperativos, pois a evolução da doença é invariavelmente fatal se uma terapia adequada não for prontamente adotada ${ }^{(1,3,4)}$. Com este intuito, o método tradicionalmente empregado era a drenagem cirúr- gica $^{(1,3,5,6)}$, geralmente associada a índices significativos de morbidade e mortalidade (10-47\%), sendo necessária anestesia geral, além de ser, por vezes, de difícil realização, por causa da localização profunda, multiplicidade e reduzido volume de alguns abscessos ${ }^{(3,7,8)}$.

O desenvolvimento e disseminação de técnicas radiológicas diagnósticas e terapêuticas têm permitido uma nova abordagem das coleções hepáticas. A avaliação radiológica, preferencialmente por 
técnicas seccionais como ultra-sonografia (US), tomografia computadorizada (TC) e ressonância magnética (RM), permite melhor visibilização da coleção e das suas relações com os espaços anatômicos do que aquela obtida por técnicas convencionais e cirúrgicas ${ }^{(\mathbf{7 , 9 , 1 0})}$. Além disso, estes métodos orientam procedimentos intervencionistas, como a punção aspirativa (PA) e a drenagem percutânea (DP), alternativas satisfatórias à cirurgia convencional, com taxas semelhantes ou superiores de sucesso, mas com menores índices de morbidade, mortalidade e complicações ${ }^{\mathbf{( 9 , 1 1 - 1 4 )}}$.

São raros os artigos que comparam os resultados obtidos no tratamento das coleções com as diferentes modalidades de drenagem ${ }^{(13,15)}$. A DP é método consagrado no tratamento das coleções hepáticas $^{(2,7,10,13,15,16)}$, pois permite controle da drenagem por manutenção de drenos. Por outro lado, apesar de ser um método simples, seguro e rápido, a PA não ganhou popularidade nos últimos anos, sendo considerada, por muitos, apenas um procedimento diagnóstico ${ }^{(16,17)}$. Alguns trabalhos recentes, no entanto, têm encorajado a utilização da PA, salientando eficiência equivalente e custos menores em relação à $\mathrm{DP}^{(13,18,19)}$.

Realizamos este estudo prospectivo com o intuito de verificar e comparar a eficácia da PA e da DP, orientadas por métodos de imagem, no tratamento das coleções hepáticas.

\section{MATERIAIS E MÉTODOS}

No período de março de 1996 a julho de 1998, foram encaminhados 52 pacientes ao Departamento de Diagnóstico por Imagem da Universidade Federal de São Paulo/Escola Paulista de Medicina, para tratamento percutâneo de coleções intra-abdominais, segundo indicação da equipe clínico-cirúrgica do Hospital São Paulo.

Destes 52 pacientes, 17 apresentavam coleções hepáticas. Os sintomas mais comuns eram febre, dor abdominal, náuseas e vômitos. Foram observadas oito coleções como complicações pós-operatórias (apendicectomia, colecistectomia, colectomia), uma como complica- ção de biópsia hepática, uma como complicação de endocardite e sete coleções em grupos etários extremos, imunodeprimidos e alcoólatras.

A maioria das coleções era piogênica, não havendo registros de abscessos amebianos dentre as avaliadas. Foram excluídas do estudo as coleções rotas.

Dos 17 pacientes, dez $(58,8 \%)$ eram do sexo masculino e sete $(41,2 \%)$ eram do sexo feminino, com média de idade de 41,6 anos, variando de oito a 92 anos.

O diagnóstico das coleções foi confirmado por intermédio de US e/ou TC. A orientação dos procedimentos percutâneos foi realizada nos equipamentos de ultra-som modelos Tosbee ${ }^{\circledR}$ (Toshiba) e HDI Ultramark- $9^{\circledR}$ (ATL).

Os critérios para a realização do procedimento incluíram presença de coleção definida por TC ou US, ausência de resposta ao tratamento clínico com antibioticoterapia, via de acesso segura para a realização do procedimento e consentimento do paciente. A escolha do procedimento foi determinada pelo médico responsável pelo paciente, sem a participação ou interferência do radiologista.

Os procedimentos percutâneos foram realizados por dois radiologistas com experiência intervencionista, os quais informaram aos pacientes os riscos e benefícios do procedimento. Foram considerados aptos para o tratamento percutâneo aqueles pacientes com número de plaquetas superior a $50.000 / \mu 1$.

$\mathrm{O}$ volume das coleções estudadas foi calculado pela fórmula da elipse (volu$\mathrm{me}=\mathrm{L} \times \mathrm{T} \times \mathrm{AP} \times 0,523)$, variando de 5 a $1.000 \mathrm{ml}$, com média de 293,8 $\mathrm{ml} \mathrm{e}$ mediana de $250 \mathrm{ml}$. Das coleções avaliadas, 5/17 (29,4\%) apresentavam volume inferior a $100 \mathrm{ml}, 5 / 17(29,4 \%)$ tinham volume entre 100 e $250 \mathrm{ml}$ e $7 / 17$ $(41,2 \%)$, volume superior a $250 \mathrm{ml}$.

Considerando-se as características morfológicas, 13/17 (76,5\%) das coleções eram não-complexas e 4/17 $(23,5 \%)$ eram complexas, ou seja, tratavam-se de coleções múltiplas, multiloculadas ou associadas a fístulas.

A PA foi realizada em $7 / 17(41,2 \%)$ das coleções e a DP, em 10/17 (58,8\%). Todos os pacientes fizeram uso de an- tibioticoterapia antes e após o procedimento.

Para a realização da PA foram utilizadas agulhas de punção com calibre variando de 14 a 22 G, após anestesia local com lidocaína a $1 \%$ e, quando necessário, sedação por via oral (Diazepam $^{\circledR} 10 \mathrm{mg}$ ). Iniciava-se o procedimento aspirando-se a coleção e comparandose o volume aspirado com o calculado previamente. O objetivo deste procedimento era aspirar pelo menos $50 \%$ do volume original da coleção hepática. Um pequeno volume do material aspirado (5 a $10 \mathrm{ml}$ ) era então encaminhado para análise laboratorial (cultura e pesquisa do microorganismo).

$\mathrm{Na}$ realização da DP, após anestesia local com lidocaína a $1 \%$ e sedação por via oral (Diazepam ${ }^{\circledR} 10 \mathrm{mg}$ ) quando necessário, utilizaram-se agulhas de punção com calibre variando de 14 a $22 \mathrm{G}$, com aspiração de 5 a $10 \mathrm{ml}$ do conteúdo da coleção e envio para análise laboratorial. Então, procedia-se à introdução do cateter de drenagem (8 a $12 \mathrm{~F}$ ), segundo a técnica de Seldinger ou introdução de trocarte.

$\mathrm{O}$ radiologista envolvido na execução do procedimento realizou o acompanhamento clínico-laboratorial do paciente e o controle da drenagem, com lavagens diárias do dreno com 5 a $10 \mathrm{ml}$ de soro fisiológico a $0,9 \%$ e aspiração. $\mathrm{O}$ dreno era retirado assim que o débito de drenagem atingia valores inferiores a $5 \mathrm{ml}$ em 24 horas, por dois dias consecutivos, com o paciente apresentando boa evolução clínica e laboratorial e com normalização da temperatura e do hemograma. Nos casos de evolução desfavorável, realizavam-se controles radiológicos.

Os resultados da evolução clínica dos pacientes submetidos aos métodos percutâneos foram classificados em:

- Sucesso: Resolução completa da coleção e melhora do quadro clínico e laboratorial do paciente, sendo este acompanhado durante até um ano (Figuras 1, 2 e 3).

- Falha: Ausência de preenchimento dos critérios de sucesso (Figura 4).

Optamos pela não-realização de testes estatísticos, já que a simples obser- 

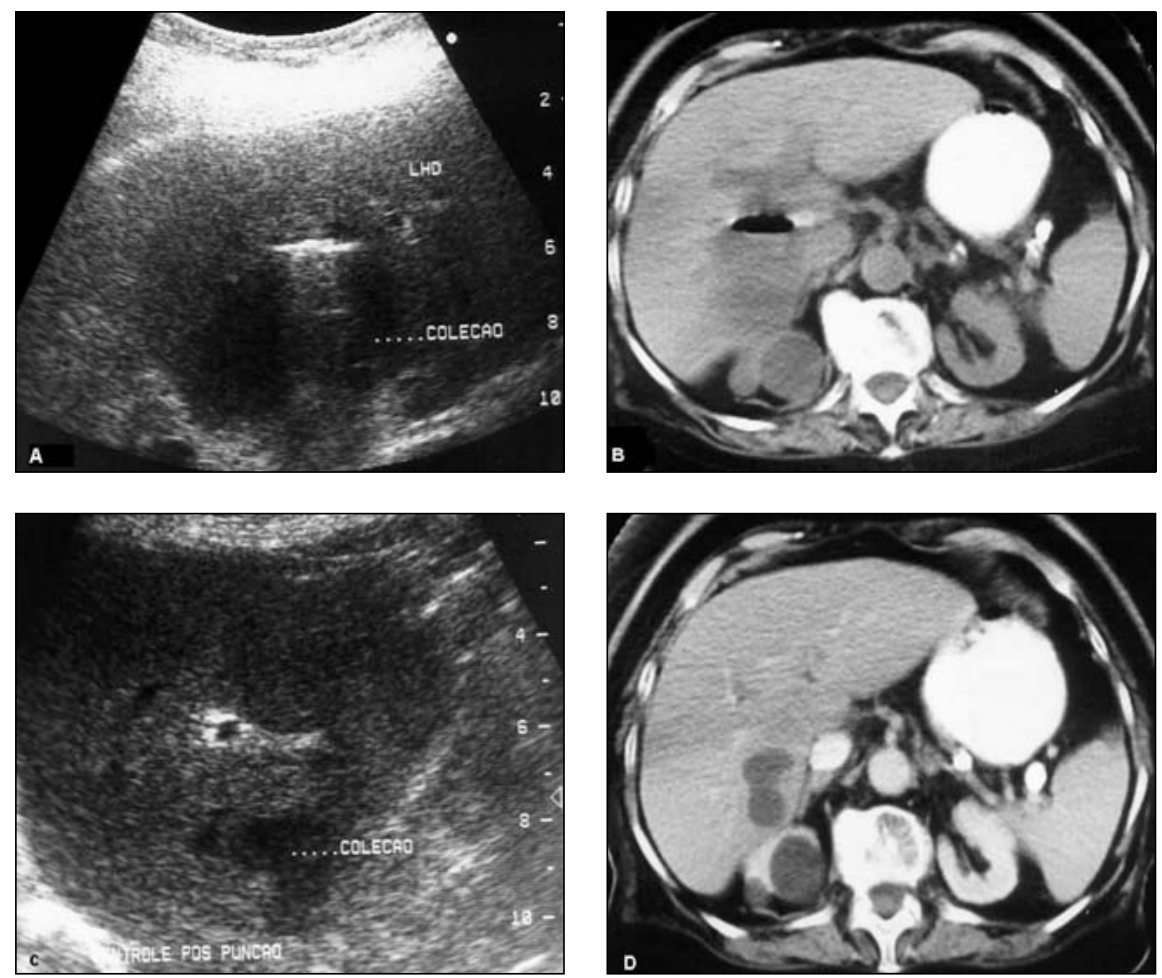

Figura 1. A,B: Coleção não-complexa submetida a punção aspirativa. C,D: Controle por US e TC, notando-se redução do volume da coleção e indicando sucesso do procedimento.
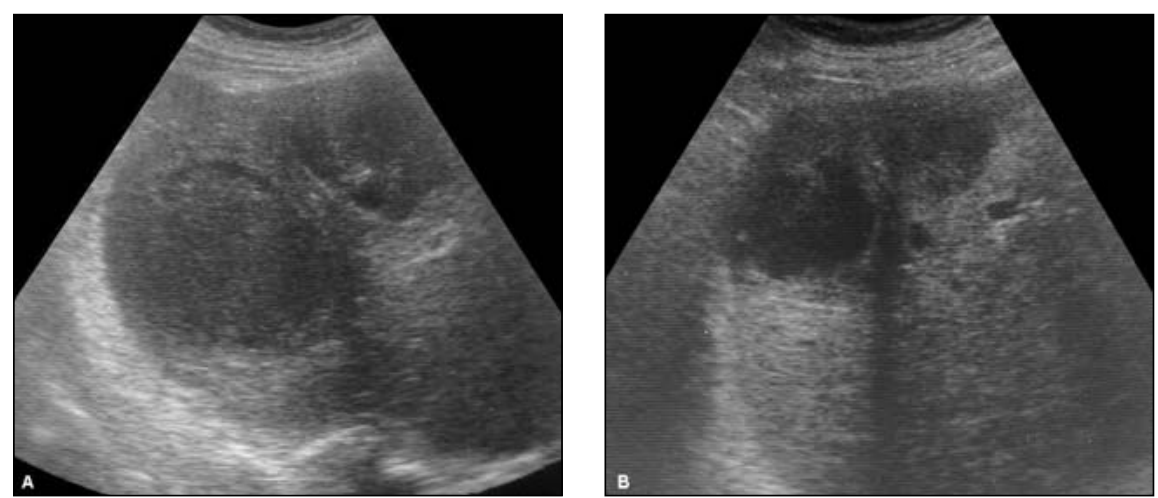

Figura 2. A: Coleção não-complexa submetida a drenagem percutânea. B: Controle por US evidencia redução das dimensões da coleção e sucesso do método.
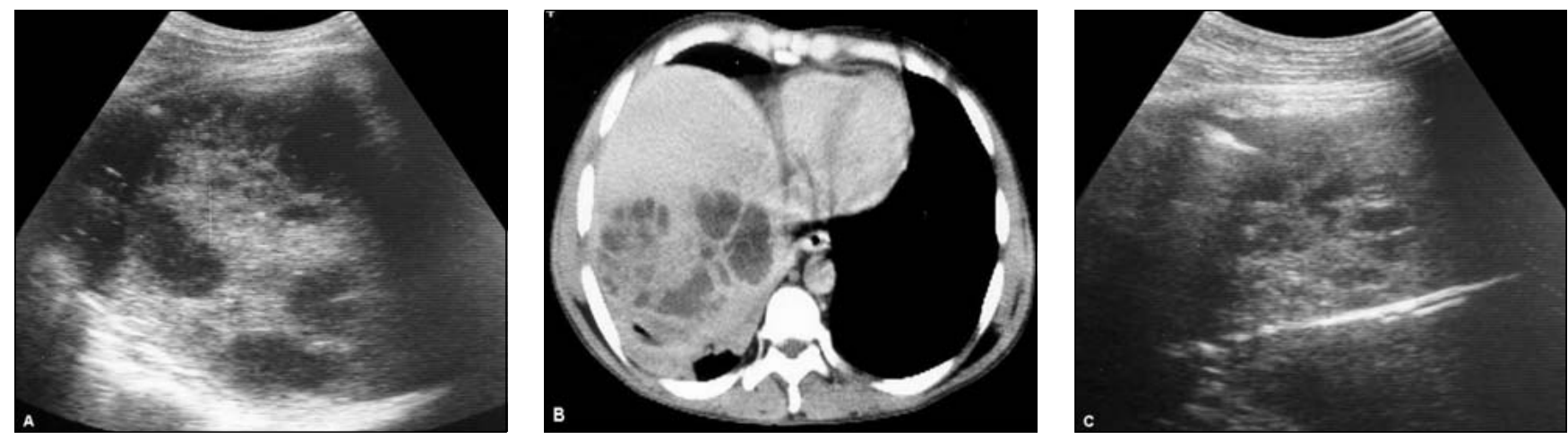

Figura 3. A,B: Coleção complexa submetida a drenagem percutânea. C: Drenagem da coleção com sucesso, notando-se redução do seu volume e melhora clínica do paciente. 

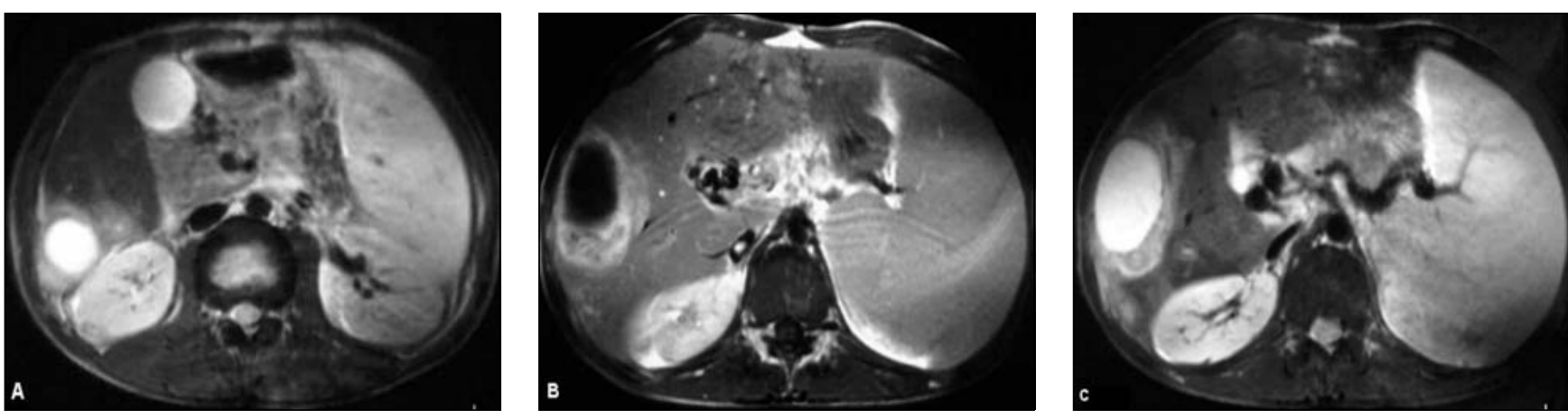

Figura 4. A,B: Paciente portador de endocardite bacteriana, com múltiplos abscessos hepáticos submetidos a punção aspirativa. C: RM de controle, notando-se recidiva dos abscessos, caracterizando falha do método percutâneo.

Tabela 1 Resultado do tratamento das coleções hepáticas, segundo o tipo de procedimento.

\begin{tabular}{|l|c|c|c|}
\hline \multirow{3}{*}{ Resultado final } & \multicolumn{3}{|c|}{ Procedimento } \\
\cline { 2 - 4 } & PA & $\begin{array}{c}\text { DP } \\
\mathrm{N}^{\circ}(\%)\end{array}$ & $\begin{array}{c}\text { Total } \\
\mathrm{N}^{0}(\%)\end{array}$ \\
\hline Sucesso & $4(\%)$ & $10(100,0)$ & $14(82,4)$ \\
Falha & $3(57,1)$ & $0(0,0)$ & $3(17,6)$ \\
\hline Total & $7(100,0)$ & $10(100,0)$ & $17(100,0)$ \\
\hline
\end{tabular}

$P A$, punção aspirativa; DP, drenagem percutânea.

Tabela 2 Resultado do tratamento das coleções com volume menor ou igual a $250 \mathrm{ml}$, segundo 0 tipo de procedimento.

\begin{tabular}{|l|c|c|c|}
\hline \multirow{2}{*}{ Resultado final } & \multicolumn{3}{|c|}{ Procedimento } \\
\cline { 2 - 4 } & $\begin{array}{c}\text { PA } \\
\mathrm{N}^{\circ}(\%)\end{array}$ & $\begin{array}{c}\text { DP } \\
\mathrm{N}^{\circ}(\%)\end{array}$ & $\begin{array}{c}\text { Total } \\
\mathrm{N}^{\circ}(\%)\end{array}$ \\
\hline Sucesso & $4(57,1)$ & $3(100,0)$ & $7(70,0)$ \\
Falha & $3(42,9)$ & $0(0,0)$ & $3(30,0)$ \\
\hline Total & $7(100,0)$ & $3(100,0)$ & $10(100,0)$ \\
\hline
\end{tabular}

PA, punção aspirativa; DP, drenagem percutânea.

Tabela 3 Resultado do tratamento das coleções com volume maior que $250 \mathrm{ml}$, segundo o tipo de procedimento.

\begin{tabular}{|l|c|c|c|}
\hline \multirow{2}{*}{ Resultado final } & \multicolumn{3}{|c|}{ Procedimento } \\
\cline { 2 - 4 } & $\begin{array}{c}\text { PA } \\
\mathrm{N}^{\circ}(\%)\end{array}$ & $\begin{array}{c}\text { DP } \\
\mathrm{N}^{\circ}(\%)\end{array}$ & $\begin{array}{c}\text { Total } \\
\mathrm{N}^{\circ}(\%)\end{array}$ \\
\hline Sucesso & $0(0,0)$ & $7(100,0)$ & $7(100,0)$ \\
Falha & $0(0,0)$ & $0(0,0)$ & $0(0,0)$ \\
\hline Total & $0(0,0)$ & $7(100,0)$ & $7(100,0)$ \\
\hline
\end{tabular}

$P A$, punção aspirativa; $D P$, drenagem percutânea.

encontrados nas coleções com volume entre 100 e $250 \mathrm{ml}$, observamos maior índice de sucesso $(75,0 \%$ vs. $33,3 \%)$ nas coleções menores $(<100 \mathrm{ml})$ do que nas de maior volume (entre 100 e $250 \mathrm{ml}$ ) (Tabelas 4 e 5).

Da mesma forma, no intuito de avaliarmos possível tendência a maior índice de sucesso, com a punção aspirativa, de acordo com a morfologia das cole- cesso em todas $(100 \%)$ as coleções nãocomplexas e na única coleção complexa (fístula com árvore biliar) drenada. Houve falha com a PA em uma coleção nãocomplexa, com volume de $100 \mathrm{ml}$, e em duas complexas, com volumes de $10 \mathrm{e}$ $250 \mathrm{ml}$ (Tabelas 6 e 7).

\section{DISCUSSÃO}

Nos últimos anos, os procedimentos percutâneos guiados por imagem têmse tornado os métodos de escolha no tratamento das coleções hepáticas, pelo menor índice de morbi-mortalidade em relação às técnicas cirúrgicas ${ }^{\mathbf{( 9 , 1 1 - 1 4 )}}$. Entretanto, a cirurgia não pode ser descartada, devendo ser considerada a alternativa terapêutica quando não há acesso percutâneo seguro, nas coleções multiloculadas ou múltiplas que exijam debridamento manual e nas falhas do pro-

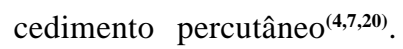

Em relação à técnica de abordagem percutânea, poucos são os artigos, na literatura, que comparam os resultados obtidos com a PA e a $\mathrm{DP}^{(\mathbf{1 3}, 15)}$. A indicação do método (drenagem ou punção) baseia-se principalmente na localização, volume e natureza da lesão. A PA apresenta vantagens por ser um procedimento menos invasivo, mais simples, barato e rápido, com menos desconforto ao paciente e exigindo menor controle médico e de enfermagem em relação à $\mathrm{DP}^{(2,18)}$, sendo ainda que múltiplas cavidades podem ser aspiradas no mesmo procedimento $^{(\mathbf{1 3})}$. Alguns autores relatam que o sucesso da PA nas coleções parenquimatosas é explicado pela maior irrigação do parênquima após o esvaziamento do 
Tabela 4 Resultado do tratamento das coleções com volume menor que $100 \mathrm{ml}$, segundo o tipo de procedimento.

\begin{tabular}{|l|c|c|c|}
\hline \multirow{3}{*}{ Resultado final } & \multicolumn{3}{|c|}{ Procedimento } \\
\cline { 2 - 4 } & $\begin{array}{c}\text { PA } \\
\mathrm{N}^{0}(\%)\end{array}$ & $\begin{array}{c}\text { DP } \\
\mathrm{N}^{\circ}(\%)\end{array}$ & $\begin{array}{c}\text { Total } \\
\mathrm{N}^{\circ}(\%)\end{array}$ \\
\hline Sucesso & $3(75,0)$ & $1(100,0)$ & $4(80,0)$ \\
Falha & $1(25,0)$ & $0(0,0)$ & $1(20,0)$ \\
\hline Total & $4(100,0)$ & $1(100,0)$ & $5(100,0)$ \\
\hline
\end{tabular}

PA, punção aspirativa; DP, drenagem percutânea.

Tabela 5 Resultado do tratamento das coleções com volume entre 100 e $250 \mathrm{ml}$, segundo o tipo de procedimento.

\begin{tabular}{|l|c|c|c|}
\hline \multirow{2}{*}{ Resultado final } & \multicolumn{3}{|c|}{ Procedimento } \\
\cline { 2 - 4 } & $\begin{array}{c}\text { PA } \\
\mathrm{N}^{\circ}(\%)\end{array}$ & $\begin{array}{c}\text { DP } \\
\mathrm{N}^{\circ}(\%)\end{array}$ & $\begin{array}{c}\text { Total } \\
\mathrm{N}^{\circ}(\%)\end{array}$ \\
\hline Sucesso & $1(33,3)$ & $2(100,0)$ & $3(60,0)$ \\
Falha & $2(66,7)$ & $0(0,0)$ & $2(40,0)$ \\
\hline Total & $3(100,0)$ & $2(100,0)$ & $5(100,0)$ \\
\hline
\end{tabular}

$\mathrm{PA}$, punção aspirativa; DP, drenagem percutânea.

Tabela 6 Resultado do tratamento das coleções não-complexas, segundo o tipo de procedimento.

\begin{tabular}{|l|c|c|c|}
\hline \multirow{2}{*}{ Resultado final } & \multicolumn{3}{|c|}{ Procedimento } \\
\cline { 2 - 4 } & $\begin{array}{c}\text { PA } \\
\mathrm{N}^{0}(\%)\end{array}$ & $\begin{array}{c}\text { DP } \\
\mathrm{N}^{0}(\%)\end{array}$ & $\begin{array}{c}\text { Total } \\
\mathrm{N}^{0}(\%)\end{array}$ \\
\hline Sucesso & $3(75,0)$ & $9(100,0)$ & $12(92,3)$ \\
Falha & $1(25,0)$ & $0(0,0)$ & $1(7,7)$ \\
\hline Total & $4(100,0)$ & $9(100,0)$ & $13(100,0)$ \\
\hline
\end{tabular}

$P A$, punção aspirativa; $D P$, drenagem percutânea.

Tabela 7 Resultado do tratamento das coleções complexas, segundo o tipo de procedimento.

\begin{tabular}{|l|c|c|c|}
\hline \multirow{2}{*}{ Resultado final } & \multicolumn{3}{|c|}{ Procedimento } \\
\cline { 2 - 4 } & $\begin{array}{c}\text { PA } \\
\mathrm{N}^{0}(\%)\end{array}$ & $\begin{array}{c}\text { DP } \\
\mathrm{N}^{0}(\%)\end{array}$ & $\begin{array}{c}\text { Total } \\
\mathrm{N}^{0}(\%)\end{array}$ \\
\hline Sucesso & $1(33,3)$ & $1(100,0)$ & $2(50,0)$ \\
Falha & $2(66,7)$ & $0(0,0)$ & $2(50,0)$ \\
\hline Total & $3(100,0)$ & $1(100,0)$ & $4(100,0)$ \\
\hline
\end{tabular}

PA, punção aspirativa; DP, drenagem percutânea.

abscesso, otimizando a ação do antibió$\operatorname{tico}^{(18)}$.

No trabalho de Gronvall et al. $.^{(\mathbf{1 5})}, 50$ coleções abdominais foram tratadas por via percutânea, com $82 \%$ de sucesso, não se observando diferença significativa entre a PA e a DP. O método, contudo, não ganhou popularidade nos últimos anos. Uma das razões pode ser a tendência de os cirurgiões sempre manterem cateteres de grande calibre no tratamento das coleções, ao contrário do que ocorre na aspiração percutânea ${ }^{(\mathbf{1}, 11)}$.
Embora na avaliação comparativa a DP apresente desvantagens, como alto custo, maior risco de infecção, lesão de estruturas e formação de hematomas ${ }^{(\mathbf{1 8})}$, este método permite melhor e mais prolongado manuseio das coleções, com drenagem contínua e reduzida possibilidade de esvaziamento incompleto ou reacúmulo delas ${ }^{(7,12,13,15,18)}$.

Num estudo comparativo, realizado por Rajak et al. ${ }^{(\mathbf{1 3})}$, a PA apresentou menor índice de sucesso (60\%) em comparação à DP (100\%). Resultados muito semelhantes foram encontrados no nosso estudo $(57 \%$ de sucesso para a PA e $100 \%$ de sucesso para a DP), embora com menor casuística.

O sucesso da PA na literatura varia de $79 \%$ a $100 \%{ }^{(\mathbf{1 8 , 1 9 , 2 1 )}}$. O baixo índice de sucesso relativo do nosso estudo pode estar relacionado ao fato de ter sido tentada uma única punção por coleção, ao contrário de outros autores, que insistiram no procedimento antes de contabilizar um insucesso ${ }^{(\mathbf{1 3})}$. Outras causas de falha incluíam microabscessos e abscessos múltiplos, que poderiam também não se beneficiar da DP.

Observamos, no nosso estudo, uma nítida tendência a um maior índice de falhas nas coleções mais volumosas submetidas a PA, apesar de não terem sido puncionadas coleções com volume superior a $250 \mathrm{ml}$. O índice de falhas foi significativamente maior $(66,7 \%)$ nas coleções submetidas a PA que tinham volume entre 100 e $250 \mathrm{ml}$ do que naquelas com volume inferior a $100 \mathrm{ml}$ (25,0\%). Acreditamos que coleções volumosas apresentem maior dificuldade de esvaziamento com aspiração única, necessitando de aspirações múltiplas. Estes resultados também foram obtidos por

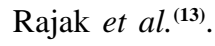

No que se refere à morfologia da coleção, notou-se tendência a um menor índice de sucesso nas coleções complexas submetidas a PA. O índice de sucesso foi significativamente maior $(75,0 \%)$ nas coleções não-complexas do que nas complexas $(33,3 \%)$. Em contraste, a DP não apresentou problemas de esvaziamento incompleto ou reacúmulo da coleção, apresentando sucesso elevado (100,0\%), à semelhança do obtido em outros trabalhos ${ }^{(13,22,23)}$.

Complicações menores foram relatadas na literatura, tanto com a DP quanto

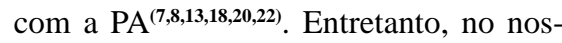
so estudo, não observamos complicações com qualquer um dos métodos, sugerindo que ambos sejam opções seguras na terapia das coleções hepáticas. É importante ressaltar, contudo, que o acesso percutâneo depende do local, do volume e da natureza da coleção, e que algumas são unicamente acessíveis à PA, pelo elevado risco de lesão de estruturas vitais. 
Uma limitação do nosso estudo é a reduzida casuística, por vezes impossibilitando a aplicação de testes estatísticos. Contudo, nosso material é homogêneo, permitindo uma comparação confiável entre os dois métodos. Maior experiência será necessária para validar estas impressões iniciais.

\section{CONCLUSÃO}

A punção aspirativa e a drenagem percutânea são métodos eficazes e seguros de tratamento das coleções hepáticas. Apesar de a PA ser mais simples, barata e rápida que a $\mathrm{DP}$, apresenta maior índice de falha, que pode ser reduzido quando se utiliza a PA em coleções menores e não-complexas. Nestes casos, a punção aspirativa é alternativa válida à drenagem percutânea na abordagem terapêutica das coleções hepáticas.

\section{REFERÊNCIAS}

1. Altemeier WA, Schowengerdt CG, Whiteley DH Abscesses of the liver: surgical considerations. Arch Surg 1970;101:258-65.

2. Berger LA, Osborne DR. Treatment of pyogenic liver abscesses by percutaneous needle aspiration.
Lancet 1982;1(8264):132-4.

3. Satiani B, Davidson ED. Hepatic abscesses: improvement in mortality with early diagnosis and treatment. Am J Surg 1978;135:647-50.

4. Sones PJ. Percutaneous drainage of abdominal abscesses. AJR 1984;142:35-9.

5. Klatchko BA, Schwartz SI. Diagnostic and therapeutic approaches to pyogenic abscess of the liver. Surg Gynecol Obstet 1989;168:332-6.

6. Mehta RB, Parija SC, Chetty DV, Smile RR. Management of 240 cases of liver abscesses. Int Surg 1986;71:91-4.

7. Gerzof SG, Johnson WC, Robbins AH, Nabseth DC. Intrahepatic pyogenic abscesses: treatment by percutaneous drainage. Am J Surg 1985;149: 487-94.

8. Lambiase RE, Deyoe L, Cronan JJ, Dorfman GS. Percutaneous drainage of 335 consecutive abscesses: results of primary drainage with 1-year follow-up. Radiology 1992;184:167-9.

9. Dondelinger RF. Advances in abdominal interventional radiology. Lancet 1999;353(suppl 1):158.

10. McDonald MI, Corey GR, Gallis HA, Durack DT. Single and multiple pyogenic liver abscesses. Natural history, diagnosis and treatment, with emphasis on percutaneous drainage. Medicine (Baltimore)1984;63:291-302.

11. Barakate MS, Stephen MS, Waugh RC, et al. Pyogenic liver abscess: a review of 10 years' experience in management. Aust N Z J Surg 1999; 69:205-9.

12. Bernardino ME, Berkman WA, Plemmons M, Sones PJ Jr, Price RB, Casarella WJ. Percutaneous drainage of multiseptated hepatic abscess. J Comput Assist Tomogr 1984;8:38-41.

13. Rajak CL, Gupta S, Jain S, Chawla Y, Gulati M,
Suri S. Percutaneous treatment of liver abscesses: needle aspiration versus catheter drainage. AJR 1998;170:1035-9.

14. Yu A, Mindelzun RE, Jeffrey RB Jr. Hepatic abscess following transhepatic drainage of subphrenic abscess. Abdom Imaging 1999;24:163-4.

15. Gronvall S, Gammelgaard J, Haubek A, Holm HH. Drainage of abdominal abscesses guided by sonography. AJR 1982;138:527-9.

16. Kuligowska E, Connors SK, Shapiro JH. Liver abscess: sonography in diagnosis and treatment. AJR 1982;138:253-7.

17. Maher JA Jr, Reynolds TB, Yellin AE. Successful medical treatment of pyogenic liver abscess. Gastroenterology 1979;77(4 Pt 1):618-22.

18. Baek SY, Lee MG, Cho KS, Lee SC, Sung KB, Auh YH. Therapeutic percutaneous aspiration of hepatic abscesses: effectiveness in 25 patients. AJR 1993;160:799-802.

19. Giorgio A, Tarantino L, Mariniello N, et al. Pyogenic liver abscesses: 13 years of experience in percutaneous needle aspiration with US guidance. Radiology 1995;195:122-4.

20. Lurie K, Plzak L, Deveney CW. Intra-abdominal abscess in the 1980s. Surg Clin North Am 1987; 67:621-32.

21. Giorgio A, Amoroso P, Francica G, et al. Echoguided percutaneous puncture: a safe and valuable therapeutic tool for amebic liver abscess. Gastrointest Radiol 1988;13:336-40.

22. Bertel CK, van Heerden JA, Sheedy PF II. Treatment of pyogenic hepatic abscesses: surgical vs percutaneous drainage. Arch Surg 1986;121:554-8.

23. Attar B, Levendoglu H, Cuasay NS. CT-guided percutaneous aspiration and catheter drainage of pyogenic liver abscesses. Am J Gastroenterol 1986; 81:550-5. 